\title{
Flow of an Oldroyd-fluid in a contracting channel with a moving boundary *)
}

\author{
R. K. Bhatnagar, M. G. N. Perera, and K. Strauß \\ Lehrstuhl für Strömungsmechanik, Abteilung Chemietechnik der Universität Dortmund (F.R.G.) \\ and Department of Mechanical Engineering, Twente University of Technology, Enschede (The Netherlands)
}

\begin{abstract}
The industrial process of coating flat surfaces with polymeric substances is numerically simulated by solving the full equations of motion for a flow through a contraction with a moving boundary. The four-constant Oldroyd constitutive equation is used to represent the viscoelastic fluid.

Some adjustments to existing finite-difference methods are made in such a way as to avoid singular iterative matrices during the solution process.

Results are presented for flow situations with Weissenberg numbers up to about three times larger than any previously published results for this problem.
\end{abstract}

Key words: Coating, planar contraction, moving boundary, Oldroyd constitutive equation, finite-difference method

\section{Introduction}

In production processes, in which a thin layer of material is coated onto a flat surface, sharp-edged plates close to the moving surface being coated are used to control the thickness of the coated layer. The materials used in coating are polymer melts and polymer solutions which are often visco-elastic in behaviour. It often happens that these coated surfaces show an irregular structure even when a smooth surface is desired. It is known that some of these effects are due to the high shear rate of the fluid between the moving surface and the fixed controlling plate.

In this paper we are especially interested in studying the effects which are due to the shear thinning and the elastic properties of the fluid. For this purpose we use the four-constant Oldroyd model as the constitutive equation.

As a first step in modelling the physical situation of the coating process we consider the flow in a planar contraction with a moving boundary as shown in figure 1 , the flow being induced by the parallel moving plane boundary. To understand the flow be-

*) Paper presented (by R. K. Bhatnagar) at the Golden Jubilee Meeting of the Society of Rheology, Boston, Oct. 29-Nov. 2, 1979.

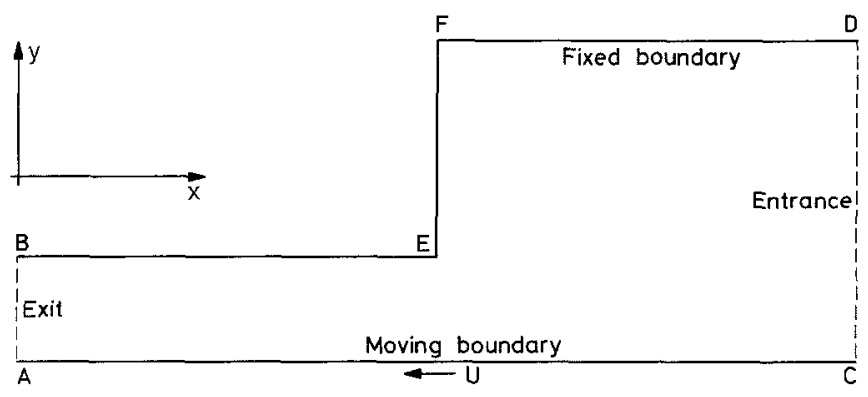

Fig. 1. Flow geometry

haviour we examine streamline patterns and velocity profiles for various fluid parameters.

\section{Formulation of the equations}

\subsection{Fundamentals}

To describe the behaviour of visco-elastic fluids with long memory effects under all possible deformations implicit rheological models have to be used [1]. Such a set of equations is provided by the so-called Oldroyd constitutive equations [2]. The complexity of the equations of motion and the necessity of taking into account the implicit nature of the constitutive 
equation require that the method of solution be numerical.

Generally, these equations can be written in integral or differential form. For the numerical solution of both forms we may utilize either a finite-difference or a finite-element approach. All methods should produce similar results. Apart from the difficulties peculiar to each method, all these methods fail, without exception, to produce proper solutions for high values of elastic parameters, that is, for Weissenberg-numbers greater than of order unity. Serious efforts have been made to overcome these difficulties $[4,6]$. By some adjustments to the existing finite-difference methods $[1,5,6]$ it has been possible in this work to obtain solutions with elastic parameters that are two or three times larger than those previously possible for the flow geometry considered [10]. The relevant adjustments and reasons for their success are described. Such modifications are generally possible, independent of the particular flow situation considered.

\subsection{Equations and boundary conditions}

In cartesian tensors the equations of motion for steady flow can be written as

$$
\begin{aligned}
& \rho u_{j} \frac{\partial u_{i}}{\partial x_{j}}=-\frac{\partial p}{\partial x_{i}}+\frac{\partial P_{i j}^{\prime}}{\partial x_{j}}, \\
& P_{i j}=-p \delta_{i j}+P_{i j}^{\prime},
\end{aligned}
$$

where $P_{i j}$ is the total stress tensor, $p$ an arbitrary isotropic pressure, $\rho$ the density and $\delta_{i j}$ the Kronecker delta. The four-constant Oldroyd constitutive equation is

$$
P_{i j}^{\prime}+\lambda_{1} \frac{\mathfrak{D} P_{i j}^{\prime}}{\mathfrak{D} t}+\mu_{0} P_{k k}^{\prime} e_{i j}=2 \eta_{0}\left[1+\lambda_{2} \frac{\mathfrak{D}}{\mathfrak{D} t}\right] e_{i j}
$$

where $e_{i j}$ is the rate of strain tensor and $\mathfrak{D} / \mathfrak{D} t$ is the so called upper convected time derivative introduced by Oldroyd [2]. When operating on a cartesian tensor this is given by

$$
\frac{\mathfrak{D} b_{i j}}{\mathfrak{D} t}=\frac{\partial b_{i j}}{\partial t}+v_{m} \frac{\partial b_{i j}}{\partial x_{m}}-\frac{\partial v_{i}}{\partial x_{m}} b_{m j}-\frac{\partial v_{j}}{\partial x_{m}} b_{i m}
$$

where $v_{i}$ is the velocity vector, $\lambda_{1}, \lambda_{2}$ and $\mu_{0}$ are material constants each with dimensions of time (with $\lambda_{1} \geqslant \lambda_{2} \geqslant 0$ and $\mu_{0} \geqslant 0$ ) and $\eta_{0}$ is the zero-shear viscosity.

The following substitution is used for the stresses before they are substituted into eq. (1):

$$
P_{i k}^{\prime}=\bar{P}_{i k}+2 \eta_{0} e_{i k} .
$$

With the pressure eliminated from eq. (1) and using eq. (5) the following equation can be obtained:

$$
\begin{aligned}
& \mu_{0} \nabla^{2} \omega+\rho\left(\frac{\partial \Phi}{\partial x} \frac{\partial \omega}{\partial y}-\frac{\partial \Phi}{\partial y} \frac{\partial \omega}{\partial x}\right) \\
& =\frac{\partial^{2}}{\partial x \partial y}\left(\bar{P}_{x x}-\bar{P}_{y y}\right)-\left(\frac{\partial^{2}}{\partial x^{2}}-\frac{\partial^{2}}{\partial y^{2}}\right) \bar{P}_{x y},
\end{aligned}
$$

where $x, y$ are cartesian coordinates (fig. 1),

$$
\Delta=\frac{\partial^{2}}{\partial x^{2}}+\frac{\partial^{2}}{\partial y^{2}}, \quad \omega=-\Delta \Phi,
$$

with $\Phi$ as the stream function satisfying the continuity equation:

$$
u=\frac{\partial \Phi}{\partial y} ; \quad v=-\frac{\partial \Phi}{\partial x},
$$

$u, v$ are velocity components.

To discuss the boundary conditions reference is made to figure 1 . The no-slip condition is assumed at all rigid boundaries. At the entrance $C D$ and at the exit $A B$ fully developed flow conditions are assumed to exist. The boundary $C A$ moves with a constant velocity $U$ in direction $C A$. At $C D$ the fully developed Couette-flow is given by a linear velocity profile, the corresponding stream function is of the form

$$
\Phi(y)=\alpha y(1-\beta y),
$$

where $\alpha$ and $\beta$ are suitable constants.

However, at $A B$ the velocity profile is not linear, but rather fully developed so that at $A B, v=0$ and therefore

$$
\frac{\partial \Phi}{\partial x}=0
$$

for all $y$.

At all fixed boundaries and regions with fully developed flow the stress equations (3) reduce to a set at algebraic equations, whose solution is straight- 
forward. On the moving boundary eq. (3) reduce to a set of ordinary differential equations, which can be solved as a two-point boundary value problem, as the stress values at the points $A$ and $C$ are known.

\section{Numerical modelling}

The numerical modelling of eqs. (3), (6) and (7) is given in detail in recent publications $[1,5]$. Eq. (6) is discretized using central differences, eq. (7) using central differences for the viscous terms and upwind differences for the convective terms. The system (3) is discretized using the upwind technique [3]. The resulting systems of difference equations have diagonally dominant iterative matrices. The succesive over-relaxation method (SOR) is used to solve the equations. For each of the variables in the iterative process the outer iterate is smoothed using the formula

$$
\gamma_{(\alpha)}^{k}=\xi_{(\alpha)} \gamma_{(\alpha)}^{k-1}+\left(1-\xi_{(\alpha)}\right) \bar{\gamma}_{(\alpha)}^{k},
$$

where $\alpha$ stands for the five different variables and $k$ refers to the $k^{\text {th }}$ outer iteration, $\left(\xi_{(\alpha)} 0 \leqslant \xi_{(\alpha)} \leqslant 1\right)$ is the smoothing parameter for the variable $\alpha$ and $\bar{\gamma}_{(\alpha)}^{k}$ is the unsmoothed $k^{\text {th }}$ inner iterative solution of the variable $\alpha$. To determine the convergence of the inner and outer iterations we use a convergence criterion at all grid points of the form

$$
\frac{\left\|\gamma_{(\alpha)}^{k+1}-\gamma_{(\alpha)}^{k}\right\|}{\left\|\gamma_{(\alpha)}^{k}\right\|}<\varepsilon_{(\alpha)},
$$

with $\alpha=1,2 \ldots, 5$.

$\varepsilon_{(\alpha)}$ is generally different for different variables as well as for inner and outer iterations (see table 1).

To obtain solutions for higher elastic parameters some modifications where made. The first of eq. (3), for example, can be written as

$$
\begin{aligned}
& A_{1} P_{x x}+\lambda_{1}\left(u \frac{\partial}{\partial x}+\frac{\partial}{\partial y}\right) P_{x x} \\
& =B_{1} P_{x y}+C_{1} P_{y y}+F_{1} .
\end{aligned}
$$

After discretization using upwinding the coefficient of $P_{x x}$ i.e. the L.H.S. of eq. (13) has the form

$$
(1+\sigma)+\lambda_{1} \frac{|u|}{h}+\lambda_{1} \frac{|v|}{h},
$$

where

$$
\sigma=\left(\mu_{0}-2 \lambda_{1}\right) \frac{\partial^{2} \Phi}{\partial x \partial y}
$$

when $A_{1}=1+\sigma$ is positive and

$$
(1+\sigma)-\lambda_{1} \frac{|u|}{h}-\lambda_{1} \frac{|v|}{h}
$$

when $A_{1}$ is negative. The coefficient of $P_{y y}$ is similar to $(14,15)$ and that of $P_{x y}$ is unity.

In the numerical solution process expressions of the form $(14,15)$ cannot be allowed to be zero. Let us examine the possibility that (14) or (15) be small or zero. Physically this means very large or infinite stresses, a condition impossible in the continuum. But during the solution process

$$
-\alpha \cdot 10^{n} \leqslant(1+\sigma) \leqslant \alpha \cdot 10^{n}
$$

is a possibility, where $n$ is related to the machine capacity to hold exponents, and $\alpha$ is any positive real number $(\alpha \leqslant 1)$. The possibility of (16) can cause expressions of the form $(14,15)$ to have infinitesimal values giving values for the corresponding stresses that are too large, causing the solution of system (3) to be unstable. A possible way of avoiding this situation is to take the terms $\sigma P_{x x}$ and/or $\sigma P_{y y}$ to the R.H.S. of the respective equations of the form (13) and give these terms values from the previous iterative solution. Theoretically the converged values are the same for all the arrangements.

It may be noted that the solution often reaches convergence in an oscillatory manner especially for the stresses. In such cases the convergence is improved by using a fairly large smoothing factor for the stresses. Most of the stability and convergence difficulties appear to originate at and around the sharp corner $E$ (fig. 1). In a solution that diverges it is found that changes of about 10 or even higher occur in the stresses whereas the stream function changes its value in the fifth decimal place. However the errors at the corner seem to remain localized. It is possible that truncation and roundoff errors play an important role especially when $\lambda_{1}$ is large. We note that for $\lambda_{1}=$ $0.5 \mathrm{sec}$ the stresses vary from 0 to $1200 \mathrm{dyn} / \mathrm{cm}^{2}$ whereas the stream function is fixed by the boundary condition and varies from 0 to 0.375 within the field of calculation.

For high $\lambda_{1}$-values good starting values are essential to obtain convergence. It is best to use the continua- 
tion method, that is, to solve the equations for progressively larger values of $\lambda_{1}$. It is interesting to note that once good starting values are found the solution could be made to converge to smaller tolerances if desired. For $\lambda_{1}=0.5 \mathrm{sec}(W e \approx 2.5)$ for example the solution was made to converge to tolerances of $10^{-6}$ for the stream function and $10^{-4}$ for vorticity and stresses. Such small tolerances are normally not necessary to decide on convergence but show the stability of the solution.

\section{Numerical results and conclusion}

The equations for the problem are solved in a dimensional form. This approach facilitates the isolation of shear-thinning viscocity effects from those associated with fluid memory. The dimensions of the channel in figure 1 are taken as follows:

$$
\begin{array}{rlrl}
A B & =0.25 \mathrm{~cm} ; & C D=0.75 \mathrm{~cm} ; \\
B E=1.0 \mathrm{~cm} ; & F D=1.0 \mathrm{~cm} .
\end{array}
$$

The plane $A C$ is taken to move with a velocity $U=$ $1 \mathrm{~cm} / \mathrm{sec}$, the contraction ratio $\overline{A B} / \overline{C D}$ is taken to $1 / 3$. Grid size $\overline{A B}$ is divided into 5 equal parts and $\overline{C D}$ into 15.

The parameters $\lambda_{1}, \lambda_{2}$ and $\mu_{0}$ are so chosen that the products $\lambda_{1} \mu_{0}$ and $\lambda_{2} \mu_{0}$ are kept constant, when $\lambda_{1}$ and $\lambda_{2}$ are changed. This is done in order that the viscosity function has the same form for all values of the elastic parameters. Specifically we have taken

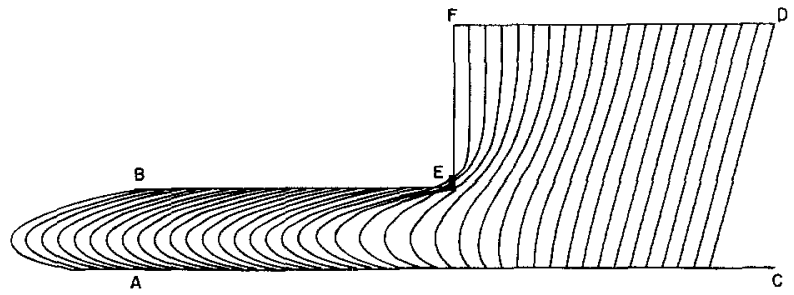

Fig. 2. Velocity profiles in channel flow direction. Scaling factor: 0.2 . Newtonian liquid $\left(\eta_{0}=5\right.$ poise)

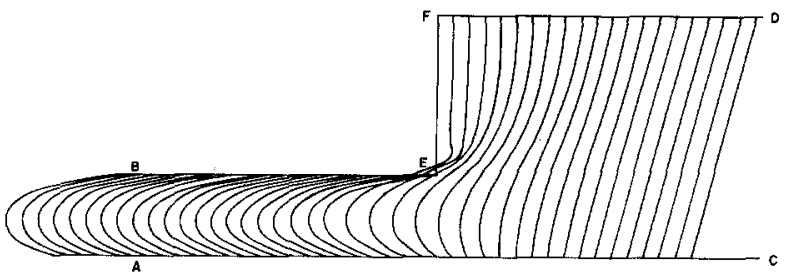

Fig. 4. Velocity profiles in channel flow direction. Scaling factor: 0.2 . Elastic liquid $\left(\lambda_{1}=0.2\right)$

$$
\begin{array}{ll}
\eta_{0}=5 \text { poise, } & \rho=1 \mathrm{~g} / \mathrm{cm}^{3}, \\
\lambda_{1} \mu_{0}=0.003 \mathrm{sec}^{2}, & \lambda_{2} \mu_{0}=0.0005 \mathrm{sec}^{2} .
\end{array}
$$

These values can be considered as fairly realistic for polymer solutions [7]. The equivalent inelastic solutions can be obtained by taking an appropriate limit. This involves making $\lambda_{1}$ and $\lambda_{2}$ progressively smaller while maintaining the products $\lambda_{1} \mu_{0}$ and $\lambda_{2} \mu_{0}$ constant [1].

Figures 2-5 depict the velocity profiles in the channel direction for a Newtonian liquid, non-Newtonian inelastic liquid and elastic liquids with $\lambda_{1}=0.2$ and $\lambda_{1}=0.5 \mathrm{sec}$ respectively, the scaling factor used in these figures is 0.2 .

As far as these velocity profiles are concerned, at least two main features are important. The first is the oscillatory behaviour of the elastic fluid in the direction of the main flow, immediately before it enters the contraction (fig. 5). This effect has already been mentioned by earlier workers $[1,5,10]$ and also been observed experimentally [11]. There is also a second sort of oscillation, namely, across the main flow direction. This is clearly seen by comparing the plots of the velocity profiles (figs. $2-5$ ) as well as in the plots which show the positions of the velocity maxima for inelastic and elastic liquids (figs. 6 and 7).

Figure 6 shows the positions of the velocity maxima for a Newtonian liquid with viscosity of 5 poise, whereas figure 7 compares the profiles of the positions of velocity maxima for a non-Newtonian inelastic and an elastic liquid with $\lambda_{1}=0.5$.

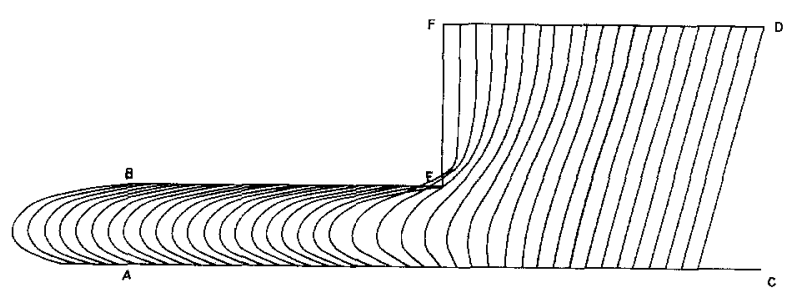

Fig. 3. Velocity profiles in channel flow direction. Scaling factor: 0.2 . Non-Newtonian inelastic liquid

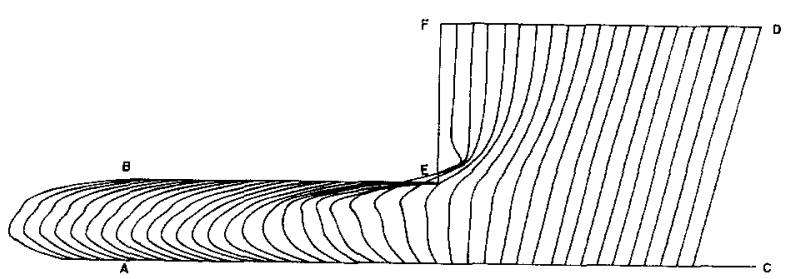

Fig. 5. Velocity profiles in channel flow direction. Scaling factor: 0.2. Elastic liquid $\left(\lambda_{1}=0.5\right)$ 
The elasticity produces distinct changes in liquid velocities near the contraction. It is seen that, for elastic liquids, higher liquid velocities exist near the rigid boundary close to the corner $E$ than for the inelastic counterpart. This gives rise to a sort of plug flow away from the corner at the entrance area. Such effects have been predicted by earlier workers and also observed in experiments $[1,5,9]$. The plots of the velocity profiles also show that the entry length is larger for elastic liquids than for Newtonian and nonNewtonian inelastic liquids.

Figure 8 shows the streamlines for a Newtonian liquid with constant shear viscosity, whereas in figures 9 to 11 we have plotted the streamlines for

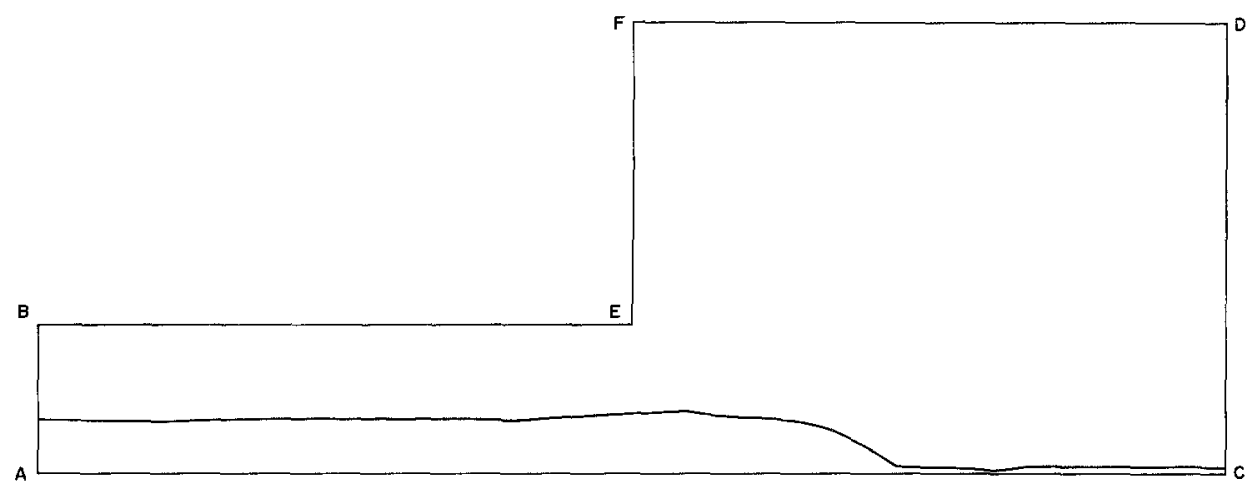

Fig. 6. Positions of velocity maxima. Scaling factor: 1.0 . Newtonian liquid $\left(\eta_{0}=5\right.$ poise $)$

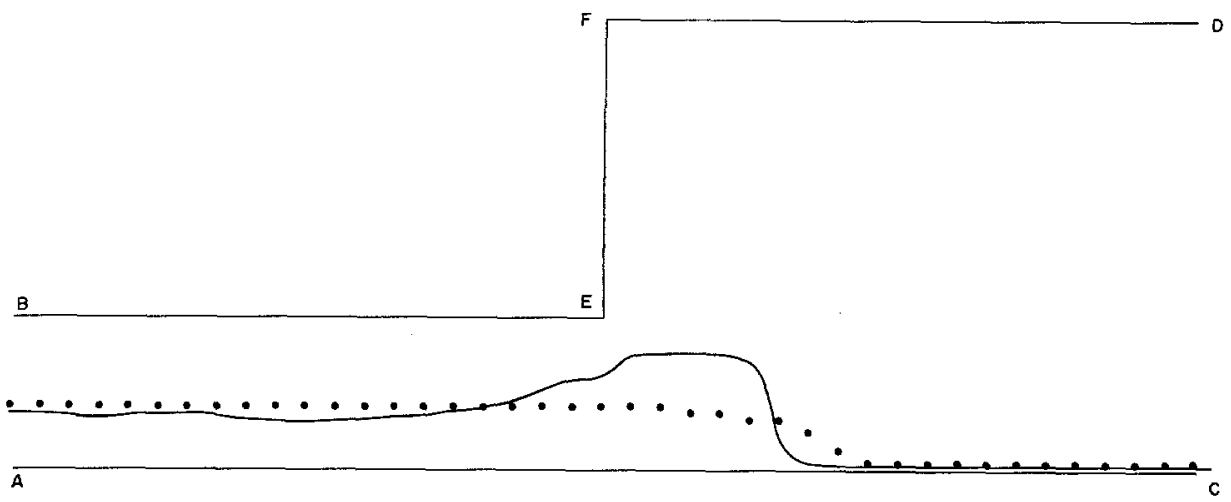

Fig. 7. Positions of velocity maxima. Scaling factor: 1.0. - Elastic liquid $\left(\lambda_{1}=0.5\right), \cdots$ non-Newtonian inelastic liquid

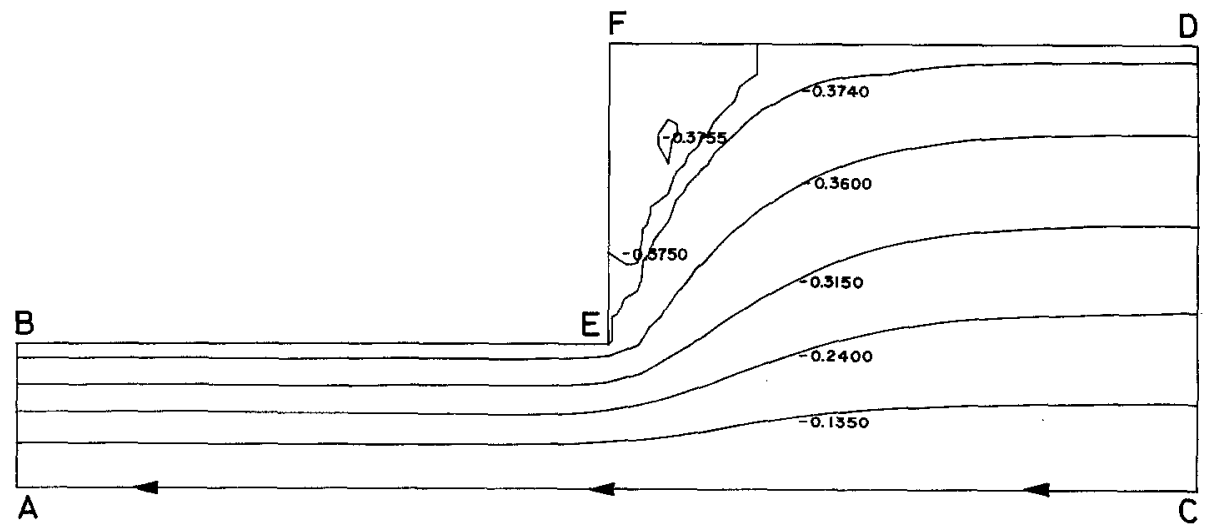

Fig. 8. Streamlines for a Newtonian liquid ( $\eta_{0}=5$ poise) 
inelastic and elastic liquids. It is interesting to make an observation regarding the recirculation area near the corner $F$. For the flow parameters utilized in the present calculations this area shows little flow activity and the size of this zone decreases with increasing elasticity. It is also seen that an increase in elasticity displaces the recirculating area away from the crosswall $E F$, towards the parallel wall $F D$ (fig. 8-11) farthest from the moving plate. In other words, with increasing elasticity the streamlines that are close to

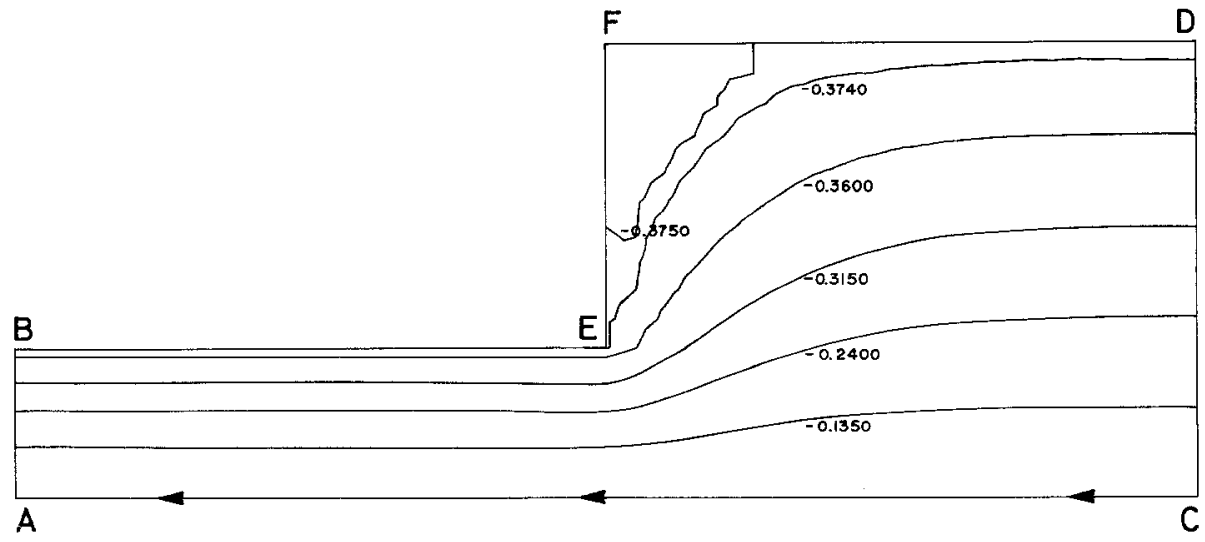

Fig. 9. Streamlines for a non-Newtonian inelastic liquid $\left(\eta_{0}=5\right.$ poise, $\left.\lambda_{1}=0.2 \mathrm{sec}, \mu_{0}=0.015 \mathrm{sec}, \lambda_{2} / \lambda_{1}=1 / 6\right)$

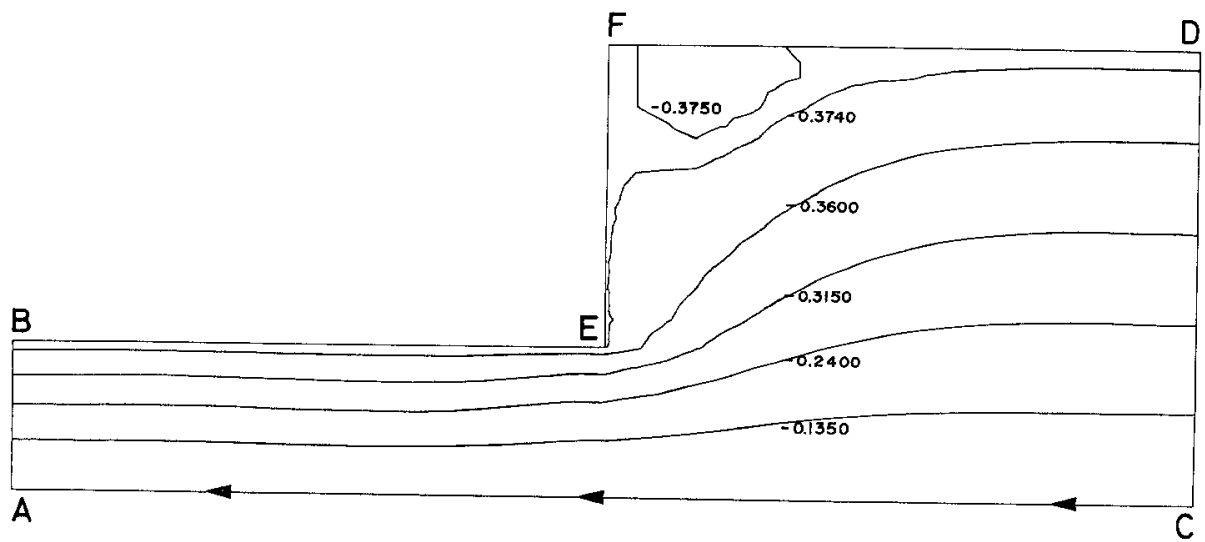

Fig. 10. Streamlines for an elastic liquid $\left(\eta_{0}=5\right.$ poise, $\left.\lambda_{1}=0.4 \mathrm{sec}, \mu_{0}=0.0075 \mathrm{sec}, \lambda_{2} / \lambda_{1}=1 / 6\right)$

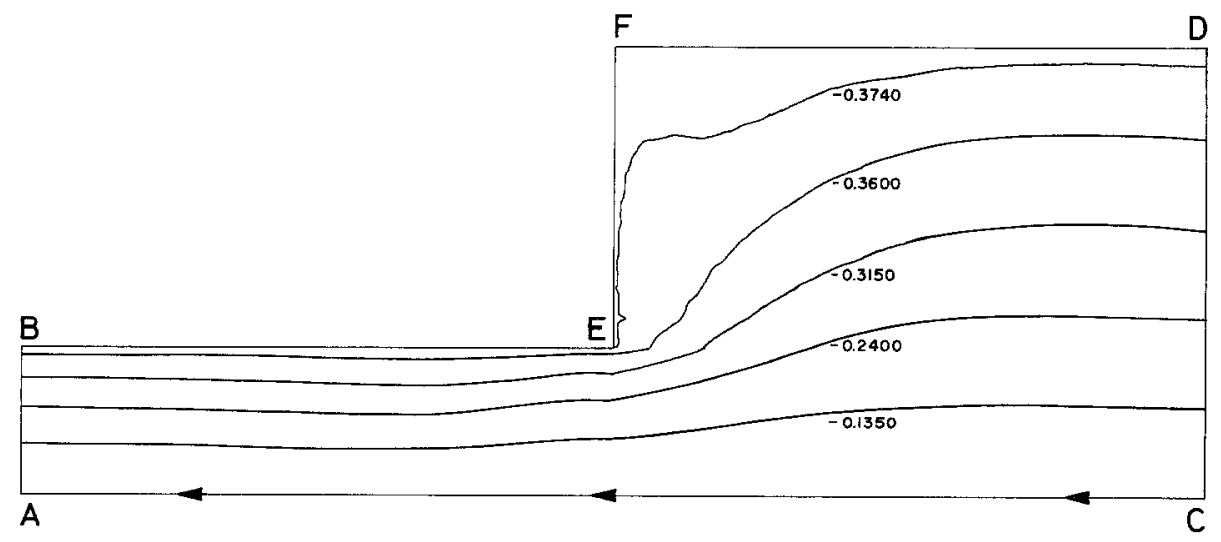

Fig. 11. Streamlines for an elastic liquid ( $\eta_{0}=5$ poise, $\left.\lambda_{1}=0.5 \mathrm{sec}, \mu_{0}=0.006 \mathrm{sec}, \lambda_{2} / \lambda_{1}=1 / 6\right)$ 


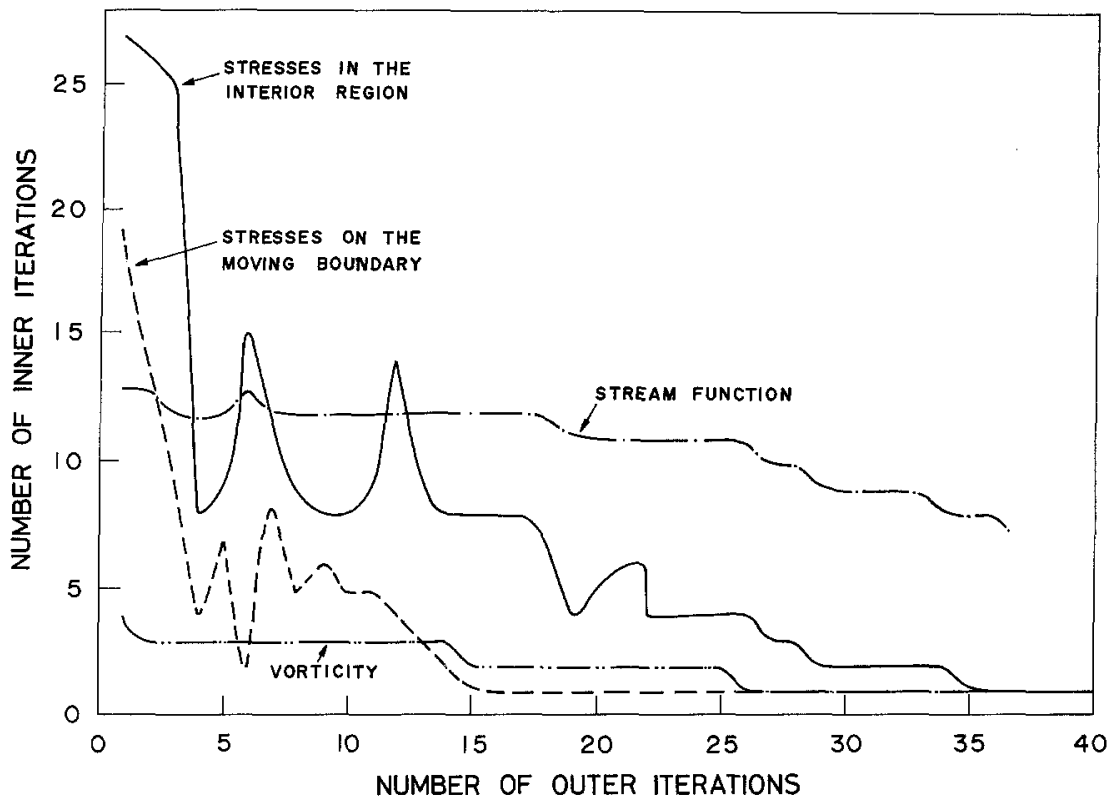

Fig. 12. Behaviour of the variables during the iterative process the wall delay their tendencies to bend towards the contraction. A similar situation is observed in experiments with convergent flows [9].

In figure 12 we have given a plot of the number of outer iterations against the number of inner iterations for stream function, vorticity, stresses in the inner region and on the moving boundary.

Efforts to obtain solutions for a higher relaxation time than $0.5 \mathrm{sec}$ were not successful in this work. As mentioned earlier divergence was due to the very high stress peaks appearing near the sharp convex corner $E$ (fig. 1). Nevertheless we were able to obtain solutions for elastic parameters (Weissenberg numbers) two to three times larger than those given in any earlier work.

\section{Acknowledgements}

This work was partly completed when the first-named author (R.K.B.) was visiting the Department of Chemical Engineering, University of Dortmund, West Germany, on an invitation from the Alexander-Von-Humboldt Foundation. This author also acknowledges the travel support given by Fundacao de Amparo a Pesquisa do Estado de Sao Paulo (FAPESP), Brasil, in January 1979.

The authors have greatly benefied from stimulating discussions with Prof. Dr. H. Giesekus. Finally, they gratefully acknowledge the computing facilities provided by the University of Dortmund and the Twente University of Technology.

\section{References}

1. Perera, M. G. N., K. Walters, J. Non-Newtonian Fluid Mech. 2, 49-61 (1977).
2. Oldroyd, J. G., Proc. Roy. Soc. A245, 278-297 (1958).

3. Greenspan, D., Lectures on the numerical solution of linear singular and non-linear differential operators, Prentice Hall (Englewood Cliffs 1968).

4. Seminar on Numerical Simulation of Viscoelastic Flows, Brown University, Providence (1979).

5. Perera, M. G. N., K. Strauß, J. Non-Newtonian Fluid Mech. 5, 269-283 (1979).

6. Chrochet, M. J., G. Pilate, J. Non-Newtonian Fluid Mech. 1, 247-258 (1976).

7. Bird, R. B., R. Armstrong, O. Hassager, Dynamics of Polymeric Liquids, Vol. I, J. Wiley and Sons (New York 1977).

8. Metzner, A. B., J. L. White, AIChE J. 11, 989 (1965).

9. Strauß, K., R. Kinast, Colloid \& Polym. Sci. 252, $753-758$ (1974).

10. Davies, A. R., K. Walters, M. F. Webster, J. NonNewtonian Fluid Mech. 4, 325- 344 (1979).

11. Busby, E. T., MacSporran: J. Non-Newtonian Fluid Mech. 1, $71-82$ (1976).

(Received March 25, 1981; in revised form December 9, 1982)

Authors' addresses:

Prof. Dr. R. K. Bhatnagar

Universidade Estadual de Campinas

Instituto de Matemática, Estatica, e Ciência da Computação 13100 Campinas, S.P. (Brasilia)

Dr. M. G. N. Perera, Prof. Dr.-Ing. K. Strauß EVT Energie- und Verfahrenstechnik GmbH

Postfach 395

D-7000 Stuttgart 1 